\title{
Le profil francisant de Ricardo Rubio et ses répercussions à l'intérieur de l'Institution Libre d'Enseignement
}

Encarnación Medina

\section{CpenEdition}

\section{Journals}

Electronic version

URL: https://journals.openedition.org/dhfles/3069

DOI: $10.4000 /$ dhfles.3069

ISSN: 2221-4038

Publisher

Société Internationale pour l'Histoire du Français Langue Étrangère ou Seconde

Printed version

Date of publication: 1 June 1999

Number of pages: p.417-435

ISSN: 0992-7654

\section{Electronic reference}

Encarnación Medina, "Le profil francisant de Ricardo Rubio et ses répercussions à l'intérieur de I'Institution Libre d'Enseignement", Documents pour I'histoire du français langue étrangère ou seconde [Online], 23 | 1999, Online since 07 July 2015, connection on 10 March 2023. URL: http:// journals.openedition.org/dhfles/3069; DOI: https://doi.org/10.4000/dhfles.3069

This text was automatically generated on 10 March 2023.

All rights reserved 


\title{
Le profil francisant de Ricardo Rubio et ses répercussions à l'intérieur de l'Institution Libre d'Enseignement
}

\author{
Encarnación Medina
}

\section{L'Institution Libre d'Enseignement}

1 À l'origine, l'Institution Libre d'Enseignement était un centre d'enseignement secondaire, aux méthodes pédagogiques actives. Ses fondateurs, Giner de los Rios, Salmerón et Azcárate, à la suite de la révocation (Guereña, 1993 : 113) de leurs chaires universitaires en 1875 , tentèrent de renouveler de l'intérieur la pratique éducative en y appliquant des méthodes modernes. «Bien vite, par ses conférences, sa bibliothèque, et surtout son Bulletin, l'Institution devint un laboratoire permanent, un lieu de rencontre obligé pour tous ceux qui concevaient l'éducation autrement et voulaient connaître d'autres expériences » (Guereña, $1997:$ 86).

2 L'Institution vient s'insérer dans le mouvement réformiste à une époque où l'influence des activités culturelles françaises - aussi bien que politiques, économiques et sociales médiatisait la vie intellectuelle de l'Espagne (Aubert, 1989 : 57-102). Une francophobie conservatrice et une francophilie libérale, considérant la France comme le pays des libertés, se partageaient le champ des intellectuels et des universitaires (Guereña, 1997: $41)$.

3 Le versant francophile dominera la rénovation des professeurs engagés dans les changements pédagogiques dont l'Espagne avait tant besoin à l'époque. Les intellectuels devinrent des figures inséparables du projet éducatif et social. Durant la Restauration, l'Institution Libre d'Enseignement fut certainement le point de départ d'un procès européisant qui proposait explicitement un rapprochement avec l'Europe 
(Guereña, 1996 : 399). Ses premières relations avec la Ligue belge de l'enseignement l'y aidèrent.

On peut difficilement imaginer un vrai débat espagnol sur l'enseignement des langues vivantes étrangères alors que l'inquiétude générale portait sur l'urgence d'un enseignement obligatoire et gratuit. A l'époque, les professeurs de l'Institution (Labra, Giner, Costa, Azcárate, Cossío, Caso, Llamas et Lledó) exigeaient du Congrès pédagogique de 1882 des écoles régies par de véritables principes pédagogiques et hygiéniques.

Depuis sa création en 1876 jusqu'en 1881, l'Institution Libre d'Enseignement vécut une première étape marquée par l'intransigeance extérieure qui fit d'elle une sorte d'Université privée. Ce fut également le moment le plus combatif. Une deuxième étape (1881-1907) commence pour le groupe réformiste quand le ministre Albareda signe le décret de restitution des professeurs à leur université. De retour à leurs chaires, l'Institution dirigera alors ses efforts vers l'enseignement primaire et le domaine qui l'identifiera jusqu'à nos jours : la réforme pédagogique. Le nouvel élan marquera les démarches éducatives de Fraebel, le rejet des examens, des manuels, de l'apprentissage théorique. Il sera temps d'implanter les créations de colonies, l'inquiétude envers la coéducation, la coopération avec les familles, les excursions, et surtout l'utilisation de la méthode socratique et de la méthode intuitive.

\section{Ricardo Rubio}

6 Né à Navalcarnero (Madrid) le 7 février 1856, Ricardo Rubio Alvarezde Linera rentre très jeune au pensionnat du collège des Escolapios de Getafe où il termine ses études secondaires. Il commence des études juridiques à l'Université de Madrid, mais, avant de finir son droit, le travail à l'Institution l'attire. Dès 1876, ayant des charges d'auxiliaire des cours de "Logique et Éthique» et de " Rhétorique et Poétique " (Jiménez-Landi, 1996 : II, 122) de Hermenegildo Giner de los Ríos, Rubio participe activement à la construction du système éducatif de l'ILE. En 1881 il est boursier au collège de Bologne en Italie. Le 20 août 1882, il retrouve à Paris Cossío rentrantd'un voyage en Suisse. Ils ont un entretien avec Solices et visitent le Musée pédagogique. A partir de ce moment, toute sa vie sera dédiée à l'Institution. Rubio meurt en 1935. Le Bulletin lui rendit hommage en y insérant des articles signés par Cossío (1935 : LIX, 97-99), Juan Ramon Jiménez (1935 : LIX, 99) et Angel do Rego (1935 : LIX, 250-251).

7 Par rapport à l'enseignement de Giner de los Rios, Rubio tient place parmi la " première génération ", intégrée par les élèves à l'Université avant 1875 et les premiers de l'Institution. Avec Rubio nous pouvons citer d'autres intellectuels dans le groupe : Manuel Bartolomé Cossío, Joaquin Costa, Eduardo Soler, Adolfo AlvarezBuylla, Leopoldo Alas Clann, Jacinto Messia, Alfredo, Laureano et Salvador Calderón, Manuel y Rafael Torres Campos, Adolfo Posada, Aniceto Sela, Pedro Dorado Montera, Rafaël Altamira, José de Caso et Luis Simarro (Abellân, 1989 : 177).

8 Les ambiances culturelles du XIX ${ }^{e}$ siècle reconnaissent en Ricardo Rubio l'écrivain, surtout le traducteur d'oeuvres de Ribot, Guyau et Fouillée. Latrentaine de traductions que nous avons pu recenser attestent un penchant philosophique et pédagogique francisant. Citons celles de G. Compayré, deRibot, de Fouillée ou de Guyau ${ }^{1}$. Les lectures, 
le travail de traducteur et les voyages le préparent à savoir écouter les innovations qui venaient d'Europe.

9 Sans données exactes sur son apprentissage du français - nous savons cependant qu'il n'assiste pas au cours de français de 1TLE, où Cossío, entre autres, assistait comme élève - nous avons cependant des informations sur ses nombreux voyages à l'étranger et surtout en France.

\section{Le Bulletin}

10 Il suffit de se reporter à l'index des noms d'auteurs collaborateurs du Boletín de la Institución Libre de Enseñanza pour se trouver face à un répertoire d'intellectuels de l'Espagne des XIX et $\mathrm{XX}^{\mathrm{e}}$ siècles. Le premier numéro du Bulletin paraît en 1877. La vocation éducative des responsables qui le dirigent fait de cette publication un instrument de dialogue de la communauté pédagogique.

Parmi les 47 articles publiés entre 1882 et 1910 par Ricardo Rubio, l'un des plus productifs collaborateurs, dans le BILE (Martinez Medrano, $1978: 21$ ), nous retiendrons ceux où l'empreinte de la pédagogie française y est le plus explicite.

En 1883, un an après la réception de l'évolution éducative en France étalée par le Bulletin avec l'article de Joaquin Costa, « La Enseñanza obligatoria en Francia » (1882 : VI, 119), Rubio publie un rapport portant sur le III $^{\mathrm{e}}$ congrès de Paris (1883 : VII, 151-152).

13 En septembre 1884 le jeune Rubio assiste au congrès international tenu au Havre. Les idées qu'il en rapporte lui serviront pour entamer une sorte de rubrique particulière qu'il nomme " Revue pédagogique ", et dont la première livraison porte sur l'enseignement primaire et professionnel à Paris (1885: IX, 219-221). En 1886, dans le même numéro où $\mathrm{M}$. Bréal signe l'article " Cómo se aprenden las lenguas extranjeras " (1886 : X, 117-121, 132-137), Rubio insère un rapport à propos du congrès de Bordeaux (1886 : X, 316-318). Il continue avec un second travail dans la direction qu'il avait initiée l'année précédente, mais en y ajoutant de plus amples lignes de mire manifestées dans le nouveau titre « Revista pedagógica extranjera » (1886:X, 79-80).

14 L'enseignement en France est au centre également d'un chapitre de son nouvel article de même titre " Revista pedagógica extranjera » (1887 : XI, 79), publié, entre autres, avec une collaboration de Sela, portant sur l'intérêt que les problèmes d'hygiène scolaire suscitaient à l'Académie de médecine de Paris (1887 : XI, 292).

Les pages du tome XII du Bulletin offrent, de la plume de Rubio, un rapport du dernier Congrès pédagogique tenu à Paris (1888 : XII, 173-177, 187-190), ainsi qu'un article envisageant la toute récente bibliothèque circulante du Musée pédagogique français (1888 : XII, 147-150, 158-160). À l'époque, les « Conversations pédagogiques », célébrées les jeudis après-midi à l'ILE et ouvertes à tout public enseignant ou étudiant, abordent des sujets aussi divers que la lecture pour enfants, les caractères des livres pédagogiques français par rapport aux livres anglais, L'éducation de la bourgeoisie de Maneuvrier ou le cours de pédagogie de Henri Marion à la Sorbonne (Jiménez-Landi, 1996 : III, 98). Rubio y participe.

16 Les observations suivies sur l'enseignement en France portent de nouveau leurs fruits en 1889 lorsque notre infatigable travailleur de l'éducation collabore avec quelques pages résumant le mouvement pédagogique français de l'année précédente (1889 : XIII, 
49-54). C'est à cette époque que José de Caso rédigea un article consacré à l'enseignement de la langue, bien que particulier à l'anglais (1889 : XIII, 339-344, 373-378). Au mois d'octobre, Rubio est à Paris. Il assiste à un cours de Pécaut. Il en revient enchanté.

Le numéro XIV du BILE insère un rapport de Lopez Selva répertoriant les congrès tenus à Paris en 1889 - d'un certain intérêt pour les pédagogues - (1890 : XIV, 177), alors que H. Marion y résume le développement des idées pédagogiques en France depuis 1870 (1890 : XIV, 214). Une année s'écoule sans collaboration de notre dévoué personnage peut-être une question de santé ; la famille Rubio attrape une forte grippe à Navalcarnero -. Ricardo Rubio travaille en 1891 à un nouveau sujet, l'enseignement de la botanique (Otero, 1994 : 256), alors que D. A. Posada propose un mémoire du Conseil général des facultés de Paris et que Gabriel Compayré publie une étude des opinions françaises de l'époque à propos de l'éducation (1891 : XV, 353-356).

Nous le retrouvons en 1897 quand il dirige toute son attention vers l'enseignement primaire à l'étranger (1897 : XXI, 9-14). Et le nouveau siècle s'inaugure pour lui avec une bourse en Allemagne. Le voyage en France de Cossío , qu'il accompagne sûrement pendant quelque temps, puis les cours de Bergson, de Durkheim, et encore les entretiens avec Boutroux, Dupuy, Guillaume, Buisson et Friedel (Jiménez-Landi, 1996 : III, 514) font pousser pour eux de nouvelles forces génératrices. Rubio s'intéresse également à la gymnastique scolaire (1908 : XXXII, 262-268) - en 1910, la Ligue belge consacra un congrès à ce sujet - ainsi qu'au laboratoire de Pédagogie de Paris (1908 : XXXII, 40-43).

19 Ce seront, cependant, les années vingt, qui seront porteuses d'une attention spéciale à l'enseignement des langues vivantes étrangères, et qui afficheront au BILE cet intérêt grâce aux travaux de Américo Castro (1921 : XLV, 119-121), de Otto Jespersen (1925 : XLIX, 321-326, 358-370 ; 1926 : L, 5-9) et de Rubén Landa (1926 : L, 35-36). En 1928, les « Institucionistas » étudient un élan possible de l'enseignement des langues vivantes à l'ILE. Ils s'inspirent des réflexions de Stanley Hall dans Some psychological aspects of teaching modem language (Otero, 1994 : 143-144) : il faut s'initier à l'âge de 10 ans; il faut connaître une langue vivante avant une langue morte ; il ne faut apprendre qu'une langue étrangère à la fois ; la deuxième ne doit venir qu'après trois années d'études ; il faut suivre la démarche phonétique de Viëtor ; il faut parler avant de lire et écrire ; et la méthode directe est insuffisante pour les étudiants adultes.

20 Pour faire justice, il nous faut insister sur l'important rôle de Rubio par rapport au Bulletin. Non seulement en tant que porte-parole d'une pédagogie française, mais aussi en tant qu'âme du BILE, puisque Giner de los Rios se décharge sur lui de toutes les responsabilités de l'édition. Et, en dehors de la publication, le fondateur de l'Institution demandait toujours l'avis de ses collaborateurs les plus directs, Cossío et Rubio.

\section{Le Musée Pédagogique National}

21 Au début de l'importante seconde étape de l'ILE, période centrée sur l'enseignement scolaire, et parmi d'autres initiatives, comme les Congrès pédagogiques, puis la Junta de Ampliación de Estudios (1907) et la Residencia de Estudiantes (1910), il faut constater que l'une des toutes premières fut le « Museo Pedagógico de Instrucción Primaria ». 
Créé en 1882, le Musée pédagogique fut placé sous la direction de Manuel B. Cossío, qui obtint le poste par concours.

À cette époque, Ricardo Rubio, dépassant son importante œuvre intellectuelle - en tant que traducteur et porte-parole des innovations pédagogiques européennes -, travaille également à des réalisations pratiques concrètes grâce à sa responsabilité au Musée pédagogique de Madrid. C'est bien à côté de son grand ami Cossío - les deux hommes avaient eu des tâches de maîtres d'école à l'Institution (Delgado Criado, 1994 : 445) qu'il fait de la pédagogie une question politique. Ils arrivent même à forcer la création d'un ministère de l'Instruction publique en 1901.

23 Le poste de secrétaire du Musée lui procurera le statut de professeur d'Ecole normale. Mais revenons en arrière, au concours qu'il lui a fallu passer avec Cossío parmi le jury. Nous insistons sur ces épreuves en raison d'une surprenante importance des langues vivantes et surtout du français. Un exercice éliminatoire, consistant en l'exposition orale d'une communication, rédigée en français, et portant sur un thème choisi par le jury, formait le premier examen - alors que l'obligation de manier la langue française n'était point exigée pour le poste de directeur du Musée, lequel n'avait à passer qu'une traduction et une conversation d'une quinzaine de minutes-. Un délai de trois heures d'isolement sans livres en conditionnait la rédaction. Puis il fallait faire une conférence sur un thème pédagogique, soit sur l'éducation, soit sur la didactique, les méthodes, les leçons de choses, etc. Troisièmement, la rédaction des observations notées à l'occasion d'une visite d'inspection dans une école primaire. Et finalement, il fallait passer une épreuve de conversation usuelle en langue française pendant trente minutes, puis une conversation en allemand, anglais ou italien, selon le choix du candidat (Rubio, Cossío, $1886: 10-14)$.

24 Le Musée fut créé par décret du 6 mai 1882 sous l'action ministérielle d'Albareda aux Travaux Publics, au numéro 7 de la rue Daoiz à Madrid, puis réglementé par l'ordre du 8 juillet 1883. Les objectifs du Musée -Musée pédagogique national depuis 1894 - visent dès sa création l'éducation pédagogique des maîtres (Cossío, Rubio, 1886) et sont, entre autres, la reproduction pour toute l'Espagne de modèles de matériel scolaire, ainsi que l'organisation de conférences publiques portant sur différentes matières de l'enseignement primaire. Les conférenciers seront obligatoirement les responsables $d u$ Musée ou des professeurs de l'École normale, mais aussi des personnalités de prestige. Plus tard, un fonds important spécialisé en éducation, puis une bibliothèque pédagogique circulante, des services de renseignements sur l'organisation de renseignement dais le pays et à l'étranger, ainsi que l'édition delivres constitueront lesaxes généraux de son programme. Toutes les démarches visaient à en terminer avec l'isolement pédagogique de notre pays par rapport à l'Europe (Garcia del Dujo, 1985).

Parmi les publications du Musée pédagogique d'Instruction primaire nousretiendrons Museo Pedagógico de instrucción primaria. Documentos pam su historia. Legislación, organización, memoria sobre sus trabajos (1886)en raison de ses deux auteurs, Manuel B.Cossío et Ricardo» Rubio. A lamême époque, Cossío publie Museo Pedagógico de instrucción primaria. Situación de la instrucción pública en Bélgica (1886), et Rubio son livreMuseo Pedagógico de instrucción primaria. La Enseñanza primaria yprofesional en Paris (1886), après avoir donné l'année précédente uneconférence insérée dans un séminaire sur l'enseignement à la maternelleDans cet ouvrage, l'auteur présente l'organisation de l'enseignement maternel, primaire élémentaire, primaire supérieur, avec descriptiondes matières - écriture et langues vivantes, morale et droit, littérature et 
histoirelittéraire, histoire générale, géographie, mathématiques, comptabilité," physique, chimie, histoire naturelle, dessin, chant, gymnastique et travauxmanuels -, ainsi que les écoles d'adultes et d'apprentis. Il informe également des institutions complémentaires, si chères aux "Instituciónitas": les caisses scolaires (pour développer l'assistance à l'école), l'inspectionmédicale des écoles, les cantines scolaires, les caisses fournisseurs du matériel et, enfin, les bourses pour les élèves des écoles primaires supérieures.

En 1890 le Musée réunit les conditions pour publier son propre catalogueprovisionnel relatif aux Archives de 1886 - , mais donnant une visionexacte de la performance du travail de Cossío, Rubio etAltarrura, même si au moment de l'impression du livre, le fonds de rétablissement était bien supérieur. Le catalogue paraîtra sous le titre Museo Pedagógico de Instrucción Primaria, Catálogo provisional (1890). Unavait-propos souligne sa fonction capitale de service à la formation des maittres ( 1890 : VII)..

Les livres du Musée - la bibliothèque universitaire la plus visitée à l'époque -, entendus aussi bien comme moyens directs de l'éducation que comme moyens indirects, sont présentés d'après l'ordre qu'ils occupent dans la fonction éducative. Des 777 ouvrages formant le fonds - en 1905, 12220 ; en 1911, 16725 -, la plupart étaient étrangers, afin d'offrir aux maîtres les livres difficiles à trouver dans d'autres bibliothèques (Otero, 1994 : 258).

En matière pédagogique, le fonds regroupait trois périodiques français : le journal hebdomadaire Manuel général de l'Instruction primaire, reçu depuis 1885 ; les exemplaires depuis 1878 de la publication mensuelle Revue pédagogique; la Revue internationale de l'Enseignement dont l'abonnement avait été pris en 1885. Les dictionnaires et encyclopédies étaient représentés par le Dictionnaire de pédagogie et d'instruction primaire (1883) de F. Buisson. L'ensemble des traités systématiques généraux contenait des ouvrages de Charbonneau, de Compayré, de Daguet, de Gerando, de Nandy et de Rousselot. En ce qui concerne les livres d'histoire de la pédagogie, nous repérons deux œuvres de H. André, plus une Histoire de la Pédagogie de Compayré, ainsi que l'Histoire de l'éducation et de l'instruction (1880) de Frédéric Dittes².

29 La section " Langage " offre une division tripartite langue maternelle/langues étrangères/littérature (Cossío, Rubio, Altamira, $1890: 85-91$ ), porteuses toutes trois d'une structuration différente. «Langue maternelle» s'articule en 'a. doctrine générale' représentée par des méthodes et des textes dont pour le français ceux de Paul Berger, de Michel Bréal et le Cours éducatif de langue maternelle à l'usage des écoles et des familles (1880-1881) en 6 volumes de Grégoire Girard ; puis la brochure Exercices préparatoires à l'enseignement grammatical, publiée à Bruxelles par la maison Lebègue, apparaît à côté de la spécification "loi ». Nous remarquons donc que quatre titres sur quatorze sont en français ${ }^{3}$, quatre en anglais et seulement six en espagnol. Sous l'épigraphe 'b. lecture et écriture' s'ouvrent deux sous-sections : 1) méthodes et 2) textes. Les " méthodes " françaises de lecture et écriture en langue maternelle dont s'enrichissait le fonds du Musée pédagogique espagnol étaient au nombre de six face aux deux titres allemands sur les dix formant le tout ${ }^{4}$. Le choix du Musée en ce qui concerne les "textes" regroupe vingt titres ${ }^{5}$ face aux dix-neuf espagnols, aux douze anglais et à un portugais.

Par contre, le français n'est pas encore parmi les « langues étrangères ». À vrai dire, seul les manuels de latin - six méthodes - et une grammaire d'allemand remplissent la section 'méthodes et textes'. En ce qui concerne les 'textes pour les traductions' le Musée n'affiche que trois titres espagnols servant à traduire les textes latins. 
Sous l'épigraphe "Littérature ", la théorie littéraire est représentée par trois traités espagnols de rhétorique et poétique. Les textes`et morceaux choisis de l'histoire de la littérature se présentent sous cinq titres dont seul le manuel allemand ${ }^{6}$ de Freund (1875) propose un tableau dédié à la littérature française.

La lacune manifeste à propos des méthodes de langues vivantes devrait pouvoir s'expliquer par une école primaire moderne encore en construction et par un essor des méthodes de français langue étrangère adaptées à la méthodologie active qui n'arrivera qu'avec la naissance du XXe siècle (Puren, 1988 : 212). Nous ne pouvons nullement chercher une négligence par rapport à la langue française du personnel responsable de la bibliothèque. Il n'y a qu'à se reporter au catalogue, sous le chapitre dédié à l'« Éducation Personnelle ", où, à part le dictionnaire de Tolhausen (1884), la littérature est longuement représentée par les 184 volumes de la Bibliothèque nationale. Collection des meilleurs auteurs anciens et modernes. Et, finalement, nous observons un certain groupement thématique autour de la vie scolaire avec des titres comme Le petit chose (1886) ou Histoire d'un écolier hanovrien (1886) ${ }^{7}$.

Rubio et Cossío imprimeront différentes brochures selon les matièresde la bibliothèque pédagogique circulante. Là, encore une fois, nous remarquons l'importance des manuels français et leur réception.Le dessin, par exemple, nous offre deux traductions : El trabajo manual en la Escuela primaria. Obra compuesta, conforme al programa oficial de laciudad de Paris, y adornada con 469 figuras intercaladas en el texte (1896) de Jullyet Rocheron (traduction de Molina Alvarez), et El ana preparatorio detrabajo manual. Traducido y adaptado del francés al castellano (1902)de R Martin (traduction de Fernándezollero).

34 Également intéressante, la bibliothèque circulante pour enfants, inaugurée le 18 février 1918 -anniversaire de la mort de Giner -, structuré en trois sections ( I, les 7-9 ans ; II, les 10-12 ans ; III, les 13-15 ans) dont le catalogue de la deuxième offre aux jeunes lecteurs des traductions deMolière, Max Nordau, Bedollière, Dumas, Feuillet, Lemmonnier, Musset, Nodier, Ourliac, Sand, etc. (ILE, 1925).

L'ancrage francisant de la vie intellectuelle et professionnelle de RicardoRubio est donc manifeste dans tous ses travaux et projets, Soit commetraducteur d'œuvres philosophiques, soit comme jeune bibliothécaire de ILE, enseignant chargé de voyager à l'étranger, maître d'école de l'Institution, responsable du Bulletin, puis, en tant que professeur d'Ecole normale, conférencier, secrétaire du Musée, cette âme dévouée affichera uneattention spéciale aux mouvements pédagogiques français, à l'espritfrançais, à la langue et à la littérature françaises. De l'intérieur même del'Institution Libre d'Enseignement, les efforts de Rubio apportèrent une tendance francisante de plus à ajouter à la rénovation pédagogique espagnole très germanisante dès son fondement. Mais, ce n'est pas seulementl'intérieur de l'ILE qui a profité de la volonté de Rubio. Le Musée était le premier levier d'influence «Institucionista » au sein de l'administration de l'État ${ }^{8}$; nous ne pourrions donc finir, par une relation transitive, sans rendre hommage à un novateur-francisant-pédagogue de notre histoire contemporaine. 


\section{BIBLIOGRAPHY}

\section{Ouvrages étudiés}

RUBIO, R. (1883), « Conclusiones del tercer Congreso de Paris », BILE, VII : 151-152.

RUBIO, R. (1885), « Revista pedagógica : la Enseñanza primaria y profesional en Paris », BILE, IX :

219-221.

RUBIO, R. (1886), Museo Pedagógico de instrucción primaria. La Enseñanza primaria y profesional en Paris, Madrid, Imprenta de M. Burgase.

RUBIO, R. (1886), « La Enseñanza industrial y comercial en el Congreso de Burdeos », BILE, X :

316-318.

RUBIO, R. (1886), « Revista pedagógica extranjera », BILE, X : 79-80.

RUBIO, R. et M. B. Cossío (1886), Museo Pedagógico de instrucción primaria. Documentos para su histôria. Législation, organizaciôn, memoria sobre sus trabajos, Imprenta de Madrid, Fortanet.

RUBIO, R. (1887), « Revista pedagógica extranjera », BILE, XI : 79.

RUBIO, R. (1888), « El Museo pedagógico de Paris », BILE, XII : 147-150, 158-160.

RUBIO, R. (1888), « El último Congreso pedagógico de Paris », BILE, XII : 173-177, 187-190.

Rubio, R. (1889), « El movimiento pedagógico francés en 1888 », BILE, XIII : 49-54.

RUBio, R., R. Altamira et M. B. Cossío (1890), Museo Pedagógico de Instrucción primaria, Catálogo provisional, Madrid, FortaneL

RUBIO, R. (1891), Museo pedagógico de Instrucción primaria. La Botánica y su Enseñanza, Madrid, Fortanet.

RUBIO, R. (1897), «La Enseñanza primaria en el extranjero, revista de1896», BILE, XXI : 9-14.

RUBIO, R. (1908), « La gimnasia escolar en Francia », BILE, XXXII : 262--268.

Rubio, R. (1908), « El laboratorio de Pedagogía normal en Paris », BILE, XXXII : 40-43.

\section{Ouvrages consultés}

ABELLÂN, J. L. (1989), Historia crítica del pensamiento español 5/1, Madrid, Espasa-Calpe.

AUBERT, P. (1989), « L'influence idéologique et politique de la France en Espagne de la fin du XIX

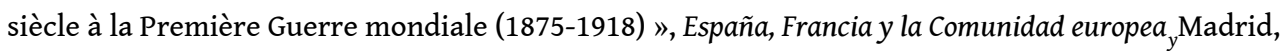
Casa de Velázquez-C.S.I.C., pp. 57-102.

Biblioteca pedagógica circulante : Reglamento y Catálogo, Madrid, Fortanet, 1889.

BRÉAL, M. (1886), « Como se aprenden las lenguas extranjeras », BILE, X : 117-121, 132-137.

CASO, J. de (1889), «La Enseñanza del idioma», BILE, XIII : 339-344, 373-378.

CASTRo, A. (1921), « La Enseñanza de las lenguas modernas », BILE, XLV : 119-121.

COMPAYRÉ, G. (1891), «La opinion contemporánea en Francia sobre la educación », BILE, XV: 353-356.

COMPAYRÉ, G. (1918), « Un pedagogo español (In memoria de Francisco Giner) », BILE , XLII : 187-188. 
cossío , M. B. (1886), Museo Pedagógico de instrucción primaria. Situación de la instrucción pública en Bélgica, Madrid, Imprenta de M. Burgase.

cossío , M. B. (1935), « Ricardo Rubio », BILE, LIX : 97-99.

COSTA, J. et al (1882), « La Enseñanza obligatoria en Francia », BILE, VI : 119.

DELGADO Criado, B. (coord.) (1994), Historia de la educación en Espana y America, vol. 3, « La educación en la Espana contemporánea (1789-1975) », Madrid, Ediciones SM.

ESTEBAN Mateo, L. (1978), Boletín de la Institución Libre de Enseñanza. Nómina bibliográfica (1877-1936), Valencia, Universidad de Valencia.

FRANçoIS, M. G. (1887), « Sobre la necesidad de hacer hablar a los discipulos en clase », BILE, XI : 93-94.

GARCIA DEL DUjo, A. (1985) : Museo Pedagógico Nacional (1882-1941). Teoria educativa y desarrollo histórico, Salamanca, Ed. Universidad.

GINER DE LOS RIOS, F. (1894), « La historia de las universidades, de M. Compayré », BILE, XVIII : 289-300, 321-28.

GUEREÑA, J.-L. (1993), « L'Université espagnole vers 1900 », SozialerRaum und akademische Kulturen. À la recherche de l'espace universitaire européen, Peter Lang, Frankfurt am Main, Berlin, Bern, New York, Paris, Wien, pp. 113-131.

GUEREÑA, J.-L. (1996) : «Infancia y escolarización », J. M. Borrás Llop (dir.), Historia de la infancia en la España contemporánea. 1834-1936, Madrid, Ministerio de Trabajo y Asuntos Sociales, Fundación Germán Sánchez Ruipérez, pp. 349-418.

GUEREÑA, J.-L. (1997) : « Les intellectuels universitaires en Espagne », M.- Chr. Granjon, N. Racine, M. Trebitsch, Histoire comparée des intellectuels, C.N.R.S., Institut d'Histoire du Temps Présent, pp. 83-90.

GUEREÑA, J.-L. (1997), «L'affaire Dreyfus en Espagne», Bulletin de la Société internationale d'histoire de l'affaire Dreyfus, $3: 41-54$.

ILE, Biblioteca circulante de niños : Catálogo de la sección segunda, Madrid, Cosano, 1925.

JESPERSEN, O. (1925), « La Enseñanza de las lenguas extranjeras », BILE, XLIX : 321-326, 358-370.

JESPERSEN, O. (1925), « La Enseñanza de las lenguas extranjeras », BILE, L : 5-9.

JIMÉNEZ, J. R. (1935), « Ricardo Rubio », BILE, LIX : 99.

JIMÉNEZ-LANDI, A. (1996), La Institución Libre de Enseñanza y su ambiente, 4 vol., « II - Periodo parauniversitario », « III - Periodo escolar 1881--1907 », Madrid, Ministerio de educación y Ciencia, Universidad Complutense de Madrid, Universidad de Barcelona, Universidad dt CastillaLa Mancha.

LANDA, R. (1926) « La Enseñanza de las lenguas vivas », BILE, L : 35-36

LÔPEZ Selva (1890), « Congresos de 1889 en Paris que interesan a la Pedagogía », BILE, XIV: 177.

MARION, H. (1890), « Desarrollo de las ideas pedagógicas en Francia desdi 1870», BILE, XIV : 241.

MARTINEZ Medrano, E. (1978), Aportaciones al estudio del Boletín de l'ILE. Análisis descriptivobibliométrico, Valencia, Universidad de Valencia.

OTERo URTAZA, E. (1994), Manuel Bartolomé Cossío : Pensamient Pedagógico y acción educativa, Madrid, Ministerio de educación Ciencia. 
POSADA, A. (1891), « Memoria del Consejo general de las Facultades deParis », BILE, XV.

PUREN, Chr. (1988), Histoire des méthodologies de l'enseignement des langues, Paris, Nathan.

R. A.[Altamira, R.] (1890), « La Enseñanza oral y los libros según Max Mulle », BILE, XIV : 292-294.

REGO, A. do (1935), « El señor Rubio », BILE, LIX : 250-251.

SELA, A. (1887), « La higiene escolar en la Academia de Medicina de París », BILE, XI : 292.

\section{NOTES}

1. Binet, A. (1929), La psicología del razonamiento, Madrid, Gráficas Faure. Bourdeau, L. (1902), El problema de la vida, Madrid. Compayré, G. (1905), La evolución intelectual y moral del niño, Madrid, Daniel Jorro. Féré, Ch., Sensación y movimiento: estudios expérimentales de psico-mecánica. Fouillée, A. (1901), Temperamento y carcter según los individuos, los sexos y las razas, Madrid, Pérez y Cfa. Fouillée, A. (1903), Bosquejo psicológico de los pueblos europeos, Granada, Fouillée, A., Fundamentos psicosociológicos de una conducta racional. Grasserie, R. de la (1904), Psicología de las religiones, Madrid, Daniel Jorro. Guyau, J.-M. (1901), Génesis de la idea de tiempo, Madrid. Guyau, J.-M. (1902), El arte desde el punto de vista sociológico, Madrid. Jardien, E. El aburrimiento. Lagrange, F. (1894), La higiene del ejercicio en los niños y en los jóvenes, Madrid. Lagrange, F. (1895), Fisiologia de los ejercicios corporales, Madrid. Ribot, Th. (1899), Las enfermedades de la memoria, Madrid, Librería de Victoriano Suárez. Ribot, Th. (1899), Las enfermedades de la personalidad, Madrid, Librería de Victoriano Suárez, Madrid, $2^{a}$ ed. 1912. Ribot, Th. (1899), Las enfermedades de la voluntad, Madrid, Librería de Victoriano Suárez, $2^{\text {a }}$ ed. 1906, $3^{\text {a }}$ ed. Faure 1922. Ribot, Th. (1899), La evolución de las ideas generales, Madrid, Librería de Victoriano Suárez, $2^{\mathrm{a}}$ ed. J. Pueyo, 1929. Ribot, Th. (1899), Psicología de la atención, Madrid, Librería de Fernando Fe, $2^{\text {a }}$ ed. 1910. Ribot, Th. (1900), La herencia psicológica, Madrid. Ribot, Th. (1900), Psicología de los sentimientos, Madrid, Librería de Victoriano Suárez. Ribot, Th. (1905), La lógica de los sentimientos, Madrid. Taine, H. (1904); La inteligencia, Madrid. Thomas, R F. (1925), La educaciôn de los sentimientos, Madrid, Imp. del Colegio nacional de sordomudos. Tissié, Ph. (1899), Lafatiga y el adiestramiento fisico, Madrid, Librería de Victoriano Suárez. Tissié, Ph. (1905), Los sueños. Fisiologia y Patologia, Madrid. Toulouse, E. (1906), Técnica de Psicología expérimental (examen de sujetos), Madrid. Villa, G., El idealismo moderno. Wepton, W. P., Principios y métodos de educación fisica e higiene.

2. André, H. (1873), Nos maîtres : hier, Paris, Hachette. André, H. (1875), Nos maîtres: aujourd'hui, Paris, Hachette. Buisson, F. (1883), Dictionnaire de pédagogie et d'instruction primaire, Paris, Hachette. Compayré, Histoire de la Pédagogie, Paris, Delaplane. Dittes, F. (1880), Histoire de l'éducation et de l'instruction, Genève, Schira-Blanchard. Gerando, B. de (1839), Cours normal des instituteurs primaires ou Directions relatives à l'éducation physique dans les écoles primaires, Paris, Renouard. Paroz, J. (1881), Histoire universelle de la Pédagogie, Paris, Delagrave. Revue pédagogique, Delagrave, Paris. Revue internationale de l'Enseignement, A. Colin, Paris.

3. Berger, $P ., U$ enseignement de la langue maternelle. Bréal, M., $U$ enseignement de la langue française.

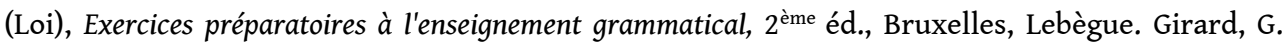
(1880-1881), Cours éducatif de langue maternelle à $V$ usage des écoles et des familles. lère', $2^{e ̀ m e}, 3^{\text {ème }}$ parties, 6 vol., Paris, Delagrave.

4. Descamps, F. (1883), Petit traité de lecture et d'élocution, à l'usage de l'enseignement, Mons, Monceaux. Dierckx, Méthode d'écriture-lecture simultanées. Livret du maître, Namur, WesmaelCharlier. Legouvé, E. (1878), El arte de la lectura, Traducción de D.J. Anchorena, con un prólogo de D.F. de A. Pacheco, Madrid, L. Polo. Legouvé, E., La lecture en action, Paris, Hetzel et Cie. Legouvé, E., La lecture en famille, Paris, Hetzel et Cie. Mirguet, V. (1882), L'enseignement de la lecture expressive à l'école primaire, Huy, Mignolet. 
5. Chervin (1881), Exercices de lecture à haute voix et de récitation, Paris, Chervin. Choix gradué de 50 sortes d'écritures pour exercer à la lecture des manuscrits, Paris, Hachette. Compayré, G., Éléments d'instruction morale et civique. Compayré, G. (1883), Lectures morales et civiques, Paris, Delaplane. Defays (1884), Méthode de lecture, d'écriture et d'orthographe à l'usage des commençants. $1^{\text {er }}, 2^{e ̀ m ' e}$ et $3^{* m e}$ livre, Liège, Dessain. Derestia, F. J. (1879), Exercices de rédaction et d'intuition, Liège, Dessain. Gallet, F. J. (1881), Méthode intuitive d'orthographe et de lecture. Livre de l'élève, Bruxelles, Lebègue. Histoire de Robinson Crusoë. Album d'images, O. Pinot, Epinal (Vosges). Jacobs, Méthode de lecture. $1^{\text {er }}$ et $2^{\text {hte }}$ livre de lecture, Bruxelles, Callewaert. Le Roy, A. (1868), L'Ami des enfants, livre de lecture, Liège, Dessain. Nouveaux petits contes pour les enfants, Strasbourg: Schultz, 1878. Pape-Carpentier, M. M. (1881), Histoires et leçons de choses pour les enfants, Paris, Hachette. Poiré, P., Simples lectures sur les principales industries. Smans, N. et E. Delelienne (1882), Lectures intuitives à l'usage des écoles et des pensionnats, Bruxelles, Manceaux. Van der Linden, Ch. (1883), Exercices simultanés de lecture et d'écriture, Namur, Wesmael-Charlier. Van Hollebeke, B. (1884), Livre de lecture. Divisions inférieure, moyenne et supérieure, Namur, Wesmael-Charlier. Villm, J. (1881), Premières lectures françaises pour les écoles primaires, Strasbourg, Schultz. Th. Hatt (1881) : Lectures enfantines. $l^{\text {ère }}$ et $2^{\text {ème }}$ parties, 2 vol., Schultz, Strasbourg.

6. Freund, W. (1875), Sechs Tafeln der Literaturgeschichte, Leipzig, W. Violet.

7. Bibliothèque nationale. Collection des meilleurs auteurs anciens et modernes, 184 vol., Paris, Berthier. Daudet, A. (1886), Le petit chose. Histoire d'un enfant, Paris, Hetzel. Dupuy, E. (1885), Les grands maîtres de la littérature russe au dix-neuvième siècle, Paris, Lecène. Laurie, A. (1886), Histoire d'un écolier hanovrien, Paris, Hetzel. Lauri, A. (1886), La vie du collège en Angleterre, Paris, Hetzel. Legouvé, E., La lecture en famille, Paris, Hetzel et Cie. Legouvé, E., La lecture en action, Paris, Hetzel et Cie. Le plus mauvais gamin du village. Imité de John Habbertcu Paris, 1884. Levoisin (1876), Tom

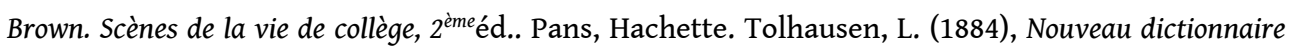
de poche espagnol-français et fiançais-espagnol, Leipzig, Tauchnitz

8. Rafael Altamira, inspecteur général pour l'enseignement primaire, est proposé pour la Direction générale. Avant tout, il se renseigne auprès de Francisco Giner de los Ríos à propos de cette nouvelle responsabilité. Giner ne lui répond qu'après un entretien avec Sela et Rubio (Jiménez-Landi, 1996 : IV, 51).

\section{AUTHOR}

\section{ENCARNACIÓN MEDINA}

Université de Cadix 